\title{
Anaesthesia Management in a Child with Rubinstein - Taybi Syndrome Scheduled for Punctoplsty
}

\author{
Ebrahim Espahhbodi, ${ }^{1,}{ }^{*}$ Hadi Ghadimi, ${ }^{1}$ and Abolfazl Kasaee ${ }^{1}$ \\ ${ }^{1}$ Farabi Hospital, Tehran University of Medical Sciences, Tehran, Iran \\ "Corresponding author: Ebrahim Espahhbodi, Anaesthesiologist, Farabi Hospital, Tehran University of Medical Science, Tehran, Iran. E-mail: eespahbodi@yahoo.com
}

Received 2017 August 14; Revised 2017 August 23; Accepted 2018 February 20.

Keywords: Rubinstein - Taybi, Anesthesia, Punctoplasty

\section{Dear Editor,}

Robinstein - Taybi Syndrome (RTS) is a rare chromosomal mutation, which was first described by Robinstein and Taybi in 1963. Leading cause of death are respiratory complications and congenital heart disease. Anaesthesia management of these patients because of a number of body systems involvement, such as heart and respiratory systems, and probability of difficult intubation due to craniofacial abnormalities are very challenging for anaesthesiologists (1). This patient was a 3 -year-old child weighting $11 \mathrm{~kg}$ with a height of $80 \mathrm{~cm}$ and came to the Operating Room (OR) for punctoplsty because of punctual atresia. He couldn't speak and walk and a neurologist diagnosed his disorder after his mental and growth retardation. Microstomia, macroglossia, and slight micrognathia were observed during physical examinations. Drug history was negative. Chest X - ray, electrocardiography, and echocardiography were normal. Lab tests were in a normal range. He was very anxious and refused to eat premedication drugs, such as ketamine or midazolam for tranquility. He didn't allow for insertion of intravenous cannulation and he tightly held his father. His father wore operating room special cloths and came with him to the OR. Because of these problems and probability of difficult intubation, it was decided to use anaesthesia with sevoflurane for inhalation induction. The researchers checked monitoring and provided difficult intubation cart and then said to his father to put on a mask over his face. Sevoflurane dial was increased step by step until $8 \%$. After the patient became deep, he was separated from his father and his father went out of the OR. Monitoring was initiated with pulse oximetry, capnography, non - invasive blood pressure examination, and electrocardiography. The researchers inserted an intravenous cannula number 20 and infused $10 \mathrm{cc} / \mathrm{kg}$ ringer lactate solution be- cause of his fasting time. Ten micro grams of fentanyl was used 3 minutes before intubation and 2 puffs of lidocaine $1 \%$ was sprayed to vocal cords during intubation. The laryngoscopic view was defined as Cormack - Lehane grade 2. After successful intubation, oropharynx was packed with wet gause and cisatracurium was injected as a muscle relaxant and sevoflurane was closed and total intravenous anaesthesia with propofol and remifentanil was started. Vital signs during surgery were stable and surgery lasted about 2 hours. At the end of surgery, self - respiration was fully recovered and reversal of cisatracurium was done by atropine and neostigmine. The patient was extubated when he began opening his eyes and he was fully awake. He was transferred to the recovery room and after one hour, no complications were found and he was transferred to the intensive care unit and discharged 2 days later.

There are many characteristics associated with RTS, such as microcephalia, beaked nose, low set ear, down slanting palpebral fissure, broad nasal bridge, strabismus, high arched eye brows, high arched and narrow palate, hypoplastic maxillary, micrognathia, microstomia, broad thumbs and hallux, poly and syndactylia, short stature, congenital heart disease, cryptorchidism, glaucoma, congenital tracheal stenosis, abnormal pulmonary lobulation, etc. (2). Sedative and opioids may increase risk of apnea in these patients and must be used carefully. Succinylcholine may produce dysrhythmia and must be used with caution (3). Difficult intubation must be predicted and alternative options have to be provided. Because of these problems and less experience about this disease, complete evaluation have to be done and more precision are necessary.

\section{References}

1. Twigg SJ, Cook TM. Anaesthesia in an adult with Rubenstein-Taybi 
syndrome using the ProSeal laryngeal mask airway. $\mathrm{Br} J$ Anaesth. 2002;89(5):786-7. doi:10.1093/bja/89.5.786.

2. Stevens's CA. RTS. Gene reviews (serial on the internet). [cited 2009 Aug].
3. Critchley LA, Gin T, Stuart JC. Anaesthesia in an infant with Rubinstein-Taybi syndrome. Anaesthesia. 1995;50(1):37-8. [PubMed: 7702143]. 